\title{
TOWARDS HIGH PERFORMANCE SMALL ANIMAL POSITRON EMISSION TOMOGRAPHY
}

\author{
Michael V. Green ${ }^{+}$, Jurgen Seidel ${ }^{+}$, Calvin A. Johnson ${ }^{+}$, \\ Juan J. Vaquero*, Javier Pascau* and Manuel Desco* \\ ${ }^{+}$National Institutes of Health, Bethesda, MD, U.S.A. \\ *Hospital Universitario Gregorio Marañón, Madrid, Spain
}

\begin{abstract}
During the last decade increasingly sophisticated positron emission tomography (PET) scanners have been developed for imaging small laboratory animals. These systems often exhibit performance characteristics, e.g. spatial resolution, substantially better than contemporary human PET scanners and are often the first systems to demonstrate new technologies, e.g. avalanche photodiodebased detector modules. Despite these advances, spatial resolution, sensitivity, resolution uniformity and other performance parameters must continue to be improved if accurate general purpose imaging is to be carried out in the most popular research subject, the mouse. Moreover, as these improvements occur, methods must also be devised to minimize the resolution-degrading effects of positron range, the distance a positron travels from the decaying nucleus before encountering and mutually annihilating an electron. Range effects are particularly important for compounds labeled with "non-traditional" positron-emitters such as $1-124$ or Tc-94m.

In order to illustrate the complex interplay of issues that must be addressed when contemplating such improvements, we describe how we have approached high performance PET imaging in the design and construction of ATLAS (Advanced Technology Laboratory Animal Scanner), a small animal PET scanner now entering service at the National Institutes of Health (NIH) in Bethesda, Md.
\end{abstract}

\section{INTRODUCTION}

Small animal (rats and mice) PET imaging is being used increasingly as a basic measurement tool in modern biomedical research. Applications of this technology occur in drug discovery and development, evaluation of therapeutic efficacy in small animal models of human disease, visualization and quantification of the site and amount of gene expression, and in many other settings, e.g. visualization and quantification of the movement of various cell types within the body.

Despite these successes, at least three important techrical issues remain to be addressed. First, the intrinsic spatial resolution of contemporary commercial [1] and research [2] small animal PET scanners is still not sufficient to allow definitive visualization of organ substructures in the mouse. Indeed, accurate quantification of radioactivity in all structures of the rat is still not possible. Many laboratories, therefore, are working to create small animal PET scanners with substantially higher intrinsic spatial resolution.

Second, in addition to improved spatial resolution, Meikle [3] and others have suggested that the sensitivity of small animal PET scanners must also be increased if pharmacologic effects are to be avoided when certain radiopharnaceuticals, e.g. receptor ligands, are administered to animais the size of the mouse. Improved sensitivity has also been identified as an important goal if iterative, resolution recovery algorithms are to be used for image reconstruction rather than conventional methods. Thus, sensitivity, as well as resolution, should be maximized.

Third, as intrinsic spatial resolution improves, the "effective" spatial resolution of a study will become increasingly dependent on positron range, particularly if isotopes other than F-18 are imaged. Positron range, the distance a positron travels from the decaying nucleus before encountering and mutually annihilating an electron, is only a fraction of a millimeter for F-18 but of the order of millimeters for other positron-emitters of biologic interest such as I-124. Since this inherent "blur" occurs in the object being imaged and is not instrument-related, no improvement in instrument performance can reduce this effect. On the other hand, if range-induced blur is ignored, the effective resolution of a study will be dominated by the radionuclide being used rather than by the imaging system. Such a result would, at the very least, complicate quantitative comparison of studies done with different radionuclides even if such measurements were made in the same animal.

These three technical issues were addressed during the research and development phase of the NIH ATLAS small animal PET scanner. In the remainder of this report, we describe how we sought to achieve simultaneously high spatial resolution, sensitivity and resolution unifonnity while providing, if not an immediate solution, at least a 
platforn for investigating potential solutions to the positron range problem.

\section{METHODS AND MATERIALS}

\subsection{Design Considerations}

Spatial resolution can be improved by reducing the crosssectional dimensions of the entrance face of the individual scintillation crystals that usually comprise ring-type PET scanners and/or by computational methods (described below). The smaller the entrance cross-section of each crystal the higher the intrinsic spatial resolution. This strategy fails when the amount of light collected from the crystal, usually from one end, is too small to allow reliable localization of the gamma ray interaction to that crystal.

Sensitivity can be increased by bringing opposing detector modules closer together (decreasing ring diameter) and by making each scintillation crystal long in the radial direction so as to present a large thickness of scintillator along the photon flight path (enhancing the probability of absorption). This strategy fails when the increase in crystal length and decrease in ring diameter causes radial spatial resolution to degrade rapidly with increasing radial distance from the geometric center of the system. This phenomena, known as the depth-of-interaction (DOI) effect, is present in all ring-type PET scanners but is particularly acute for ring diameters appropriate for small animals.

Positron range "blur" originates in the animal and not in the scanner so hardware solutions to this problem are not available. Instead, recent work has indicated that instrument-induced resolution loss and, theoretically, positron range resolution degradation can be reduced significantly by using statistical reconstruction methods that incorporate an a priori mathematical model of the imaging process. If such algorithms are provided with a model that incorporates both instrument and positron range resolution degradation, resolution losses from both of these sources should be reversible to some degree. This strategy fails when the statistical quality of the acquired image data is relatively poor (resulting in excess noise in the reconstructed images) or when the magnitude of the computational task exceeds available computing resources (a real possibility when using statistical methods).

Given this set of requirements, we deternined that the detector array should consist of "phoswich" detector modules made up of two layers of different scintillators. This strategy provides two levels of depth information that allows the detector modules to be moved closer together increasing sensitivity while reducing radial resolution degradation. At the same time, relatively long scintillator elements can be used that also increase sensitivity.

Finally, provision was made to off-load acquired data sets over the intemet to a very large scale Beowulf-type computer cluster. With this capability, image data could be reconstructed routinely with the $3 D$ ordered subset expectation maximization (OSEM) resolution recovery algorithm. When combined with the already good intrinsic spatial resolution of the scanner, effective spatial resolution should approach the mouse requirement. Thus, this computational capability was included as an integral part of the design strategy to increase spatial resolution. Accordingly, the instrument point response function is routinely included in the reconstruction algorithm for all F-1 8 studies but refinements to this model that include other effects, e.g. positron range broadening for other radionuclides, are also being studied.

\subsection{The ATLAS Small Animal PET Scanner}

The actual ATLAS scanner consists of 18 lutetium (LGSO) and gadolinium (GSO)-based phosphor-sandwich (phoswich) detector modules arranged around a ring 11.8 $\mathrm{cm}$ in diameter. Each module is comprised of a $9 \times 9$ array of $2 \mathrm{~mm}$ square $\times 15 \mathrm{~mm}$ deep phoswich elements (2.25 mm pitch), each of which is composed of a $7 \mathrm{~mm}$ long LGSO crystal optically glued end-on to an $8 \mathrm{~mm}$ long GSO crystal. The GSO end of the crystal bundle is optically glued to a miniature position-sensitive photomultiplier tube. The scintillator of-interaction (or depth) is identified by measuring the decay time (LGSO: $40 \mathrm{~ns}, \mathrm{GSO}: 60 \mathrm{~ns}$ ) of the light pulse of each event. With this technique it is possible to have a substantial total crystal depth $(15 \mathrm{~mm})$ while at the same time having (two) apparently short crystal segments.

The eighteen ATLAS detector modules are combined mechanically and electrically into groups of three to fonn a total of six sectors. The signals originating from the ATLAS sectors are fed to a data acquisition system supplied by A \& D Precision Co. (Newton, MA 02460, USA). The system consists of six charge integrating ADC modules [4] with custom modifications to facilitate identification of scintillation decay times, one scaler module, a custom coincidence logic controller, and a high speed PCI- bus interface card. The controller detects

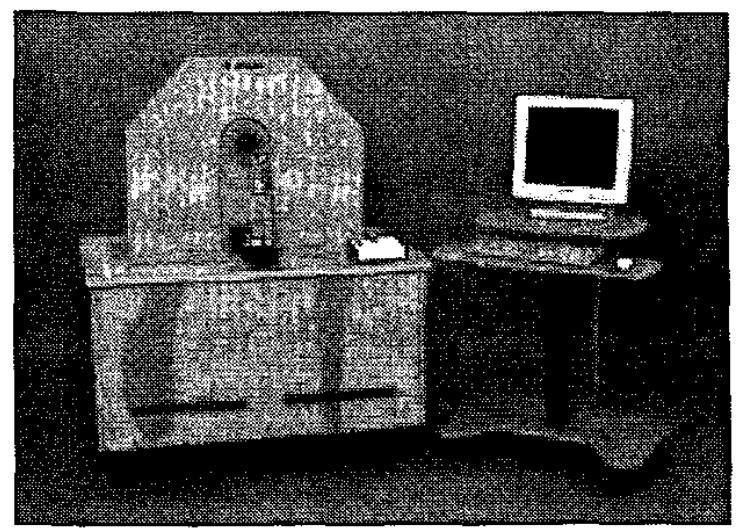

Figure 1. The NIH ATLAS small animal PET scanner. The gantry, animal imaging bed and electronics enclosure are on the left, the operator console on the right. 
coincidences between sectors and initiates signal integration and ADC read out. The data are collected by two alternating $128 \mathrm{kB}$ memory buffers on the PCI-bus card in a dual-processor PC operating under the Linux OS. The data acquisition system, power supplies, motor controllers and drivers, and computer are all housed in the wood-paneled enclosure shown in Figure 1. The aperture of the ATLAS scanner is eight-cm in diameter with a useful transverse field-of-view of six $\mathrm{cm}$ and an axial field-of-view of two cm.

The user can initiate two kinds of image reconstructions: filtered back projection on the local ATLAS computer or iterative, resolution recovery 3D OSEM reconstruction on the remote cluster. If remote reconstruction is selected, ATLAS can again be used for data collection once the acquired data set has been sent by network to the cluster. During off-site reconstruction, the status of the reconstruction is periodically updated and displayed to the user by the ATLAS user interface. When completed, the reconstructed images are automatically retumed to the ATLAS computer over the network for display and analysis.

\section{RESULTS}

\subsection{ATLAS Performance}

\section{Spatial Resolution and Resolution Uniformity}

The three major technical design goals for ATLAS were verified by measurements made on the completed system. First, ATLAS was intended to exhibit an intrinsic spatial resolution compatible with imaging animals the size of rats and mice $(<2-\mathrm{mm})$. The apparent FWHM width of a $0.5 \mathrm{~mm} \mathrm{Na}-22$ source located at the geometric center of the aperture was $1.8 \mathrm{~mm}$.

Second, radial resolution declined by $25 \%$ for a source placed $1.5-\mathrm{cm}$ off-axis and by $44 \%$ for a source $3-\mathrm{cm}$ offaxis, the edge of the effective field-of-view. The significance of these values cannot be readily judged, however, without knowing the behavior of a geometrically similar system without DOI capability. Accordingly, the variation of radial resolution with radial position for ATLAS is shown in Figure 2 compared to three simulated lutetium oxyorthosilicate (LSO) scanners with different length LSO crystals and no depth-of-interaction capability. Other than differences in crystal length, the geometry of the simulated LSO machines is identical to ATLAS. Note that ATLAS has nearly the same resolution variation as an LSO scanner with $7-\mathrm{mm}$ long LSO crystals even though the total crystal length of the ATLAS phoswich elements is $15-\mathrm{mm}$.

Central Point Source Sensitivity

Third, the system was designed to exhibit a high central point source sensitivity (CPSS) through use of relatively long phoswich crystal elements and a small ring diameter. CPSS was measured to be $1.8 \%$ for an energy window of
250-650 keV. Note that ATLAS has twice the sensitivity of the simulated 7-mm LSO scanner.

\section{D OSEM Resolution Recovery Image Reconstruction}

Figure 3 shows the result of reconstructing a data set with the 3D OSEM algorithm supplied with a Gaussian model of the instrument point response function. Each of the images shown in the Figure is of the same transaxial section of the rat head after uptake by the brain of F-18fluoro-deoxyglucose. The bright, arc-shaped structure covering the upper surface of the brain is the cortex.

The only difference between these three images is the number of iterations (or estimates) the algorithm was allowed in attempting to estimate the true activity distribution. Inspection of these images clearly demonstrates resolution improvement with increasing iteration number. Quantitative analysis also confirms that spatial resolution is better in the high iteration 3D OSEM reconstructions than in images reconstructed with filtered backprojection.

TABLE 1.

Absolute Central Point Source Sensitivity

\begin{tabular}{|c|c|c|c|c|c|}
\hline \multirow[t]{2}{*}{$\begin{array}{c}\text { Threshold } \\
\text { (keV) }\end{array}$} & \multirow[t]{2}{*}{$\begin{array}{l}\text { LSO } \\
7 \mathrm{~mm}\end{array}$} & \multirow[t]{2}{*}{$\begin{array}{c}\text { LSO } \\
10 \mathrm{~mm}\end{array}$} & \multirow[t]{2}{*}{$\begin{array}{c}\text { LSO } \\
15 \mathrm{~mm}\end{array}$} & \multicolumn{2}{|c|}{$\begin{array}{l}\text { ATLAS } 15 \mathrm{~mm} \\
\text { Phoswich }\end{array}$} \\
\hline & & & & $S^{*}$ & $\mathbf{M}^{*}$ \\
\hline 100 & $1.3 \%$ & $2.1 \%$ & $3.2 \%$ & $2.8 \%$ & $2.7 \%$ \\
\hline 250 & $0.9 \%$ & $1.5 \%$ & $2.4 \%$ & $2.0 \%$ & $1.8 \%$ \\
\hline
\end{tabular}

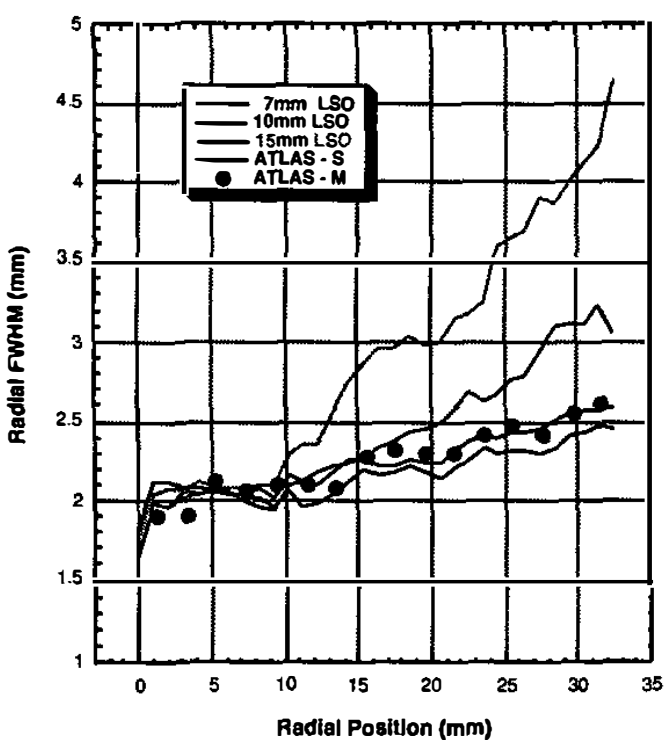

Figure 2. Radial resolution vs. radius for different scanners. ATLAS-S: simulated ATLAS results; ATLASM: measured ATLAS results. 

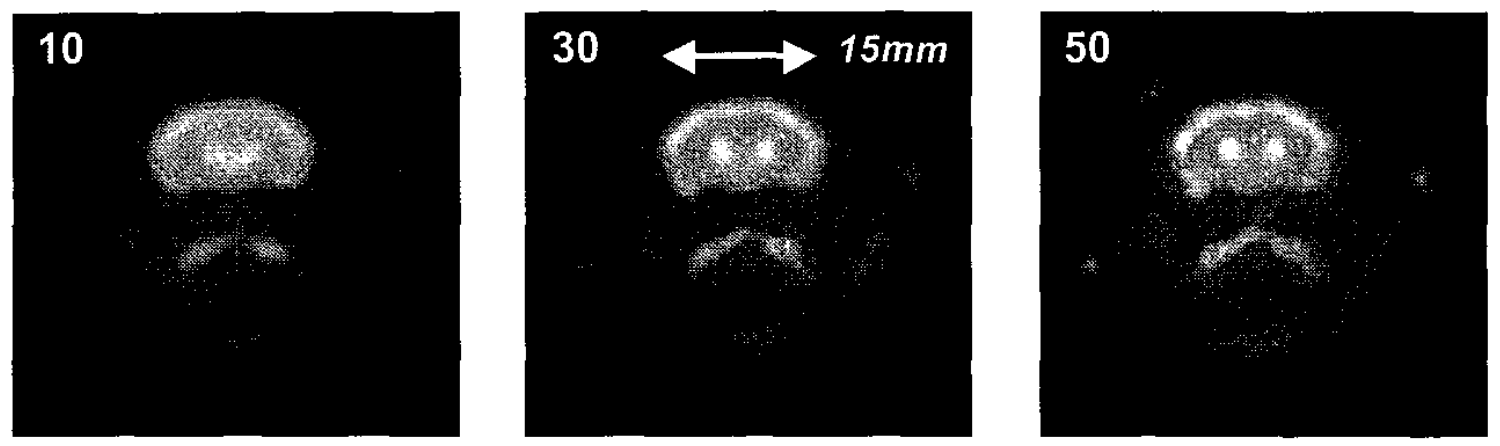

Figure 3. The effect of increasing 3D OSEM iteration number on apparent resolution in the same transverse section through the brain of a rat 30 minutes after IV administration of F-18 fluoro-deoxyglucose. The cortical rim and basal ganglia are the brightest structures in these images. Image sharpness increases with increasing iteration number: resolution at 50 iterations is approximately $1.5 \mathrm{~mm}$ compared to approximately $2 \mathrm{~mm}$ at 10 iterations. Note distance scale.

\section{DISCUSSION AND CONCLUSION}

Improvements in small animal PET require consideration of many inter-related factors. As suggested by the ATLAS example, increasing sensitivity while trying to minimize radial resolution degradation requires novel DOI-capable detector modules such as the dualscintillator, two-layer phoswich. A method to read out these modules also had to be devised in order to identify the layer-of-interaction (or depth) of an event and the position of the event across the crystal array. The degree to which this strategy is effective is illustrated in Figure 2 and Table 1. The sensitivity of ATLAS is about $33 \%$ less than an LSO scanner with 15-mm crystal length, the same length as ATLAS, but ATLAS exhibits a much smaller loss in radial resolution with increasing radius. Conversely, ATLAS possesses a radial resolution degradation nearly identical to the 7-mm LSO scanner, the crystal length of the LGSO portion of the ATLAS phoswich arrays, but is twice as sensitive. These results suggest that the use of phoswich modules is, in fact, an effective means of improving sensitivity while minimizing radial resolution degradation.

Resolution recovery algorithms offer the prospect of improving resolution beyond that obtainable with conventional filtered backprojection reconstruction [5]. Moreover, if the model supplied to the algorithm contains a faithful representation of positron range effects for the radionuclide under study as well as instrument resolution losses, it should be possible to maintain a given level of resolution for radionuclides with different, and larger, ranges. The basis for this belief is illustrated in Figure 3 where a simple Gaussian point response model representing instrument blur was supplied to the algorithm The resolution improvement observed in this example should also be attainable when range is included provided that the statistical quality of the data is high. The sensitivity requirement imposed on the ATLAS design was included precisely for this reason and data sets acquired by ATLAS for different radionuclides, e.g. I-124, Tc- $94 \mathrm{~m}$, etc. will be used to test this concept in practice.

The ATLAS project illustrates some of the issues that must be considered when building almost any high performance small animal PET scanner. As these and other obstacles to high performance small animal PET continue to be overcome, the value of this technology as a biomedical research tool will continue to grow.

\section{REFERENCES}

[1] Y. C. Tai, A. Chatziioannou, S. Siegel, J. Young, D. Newport, R.N. Goble, R. E. Nutt and S.R. Cherry, "Performance Evaluation of the microPET P4: a PET system dedicated to animal imaging", Phys. Med. Biol., vol. 46, pp. 1845-1862, 2001.

[2] A. F. Chatziioannou, S. R. Cherry, Y. Shao, R. W. Silverman, K. Meadors, T. H. Farquhar, M. Pedarsani, and M. E. Phelps, "Performance Evaluation of MicroPET: a High-resolution Lutetium Oxyorthosilicate PET Scanner for Animal Imaging", $J$ Nucl Med, vol. 40, no. 7, pp. 1164-1175, 1999.

[3] S.R. Meikle, S. Eberl, R.R. Fulton, M. Kassiou and M. J. Fulham, "The Influence of Tomograph Sensitivity on Kinetic Parameter Estimation in Positron Emission Tomgraphy Imaging Studies of the Rat Brain", Nucl Med Biol, vol. 27, pp.617-625.

[4] V. G. Zavarzin and W. A. Earle, "A 500k Event/s 12-Bit ADC System with high-speed buffered PCI Interface," in IEEE Trans Nucl Sci. vol. 46, no.3, pp. 414-416, 1999.

[5] C. A. Johnson, J. Seidel, R. E. Carson, W. R. Gandler, A. Sofer, M. V. Green, and M. E. Daube-Witherspoon, "Evaluation of 3D Reconstruction Algorithms for a Small Animal PET Camera", IEEE Trans Nucl Sci, vol. 44, no. 3, pp. 1303-1308, 1997. 